\title{
Dual-Core Photonic Crystal Fiber Plasmonic Refractive Index Sensor: A Numerical Analysis
}

\author{
Alok Kumar PAUL*, Ajay Krishno SARKAR, and Abdul KHALEQUE \\ Department of Electrical \& Electronic Engineering, Rajshahi University of Engineering \& Technology, Rajshahi-6204, \\ Bangladesh \\ *Corresponding author: Alok Kumar PAUL $\quad$ E-mail: paul10alok@gmail.com
}

\begin{abstract}
A numerical analysis on dual core photonic crystal fiber (DC-PCF) based surface plasmon resonance (SPR) refractive index sensor is presented. The guiding parameters and required sensing performances are examined with finite element method (FEM) based software under MATLAB environment. According to simulation, it is warranted that the proposed refractive index sensor offers the maximum amplitude sensitivity of 554.9 refractive index unit $\left(\mathrm{RIU}^{-1}\right)$ and $636.5 \mathrm{RIU}^{-1}$ with the maximum wavelength sensitivity of $5800 \mathrm{~nm} / \mathrm{RIU}$ and $11500 \mathrm{~nm} / \mathrm{RIU}$, and the sensor resolutions of $1.72 \times 10^{-5} \mathrm{RIU}$ and $8.7 \times 10^{-6} \mathrm{RIU}$, at analyte refractive index (RI) of 1.40 for $x$ - and $y$-polarized modes, respectively. As the sensing performance in different wavelength ranges is quite high, the proposed sensor can be used in simultaneous detection for different wavelength ranges. Therefore, the proposed device is of a suitable platform for detecting biological, chemical, biochemical, and organic chemical analytes.
\end{abstract}

Keywords: Photonic crystal fiber; biosensor; refractive index sensor; finite element method; plasmonic material

Citation: Alok Kumar PAUL, Ajay Krishno SARKAR, and Abdul KHALEQUE, "Dual-Core Photonic Crystal Fiber Plasmonic Refractive Index Sensor: A Numerical Analysis,” Photonic Sensors, 2019, 9(2): 151-161.

\section{Introduction}

A biosensor is a physical device which can be used to detect different types of biological materials [1]. Nowadays, the surface plasmon resonance (SPR) technique is being used to analyze the characteristic of the biosensor as this technique offers real-time and label-free monitoring [2]. In order to get structural contraction, photonic crystal fiber (PCF) incorporated into the SPR technique is a perfect platform of designing the biosensor instead of using prism or optical fibers because it has appealing features such as endless single-mode behavior, high nonlinearity, anomalous dispersion, controllable high birefringence, and design flexibilities (such as a number of air-holes with different diameters, and the size of pitch can be chosen depending on applications). In addition, different materials adding properties to the air-holes ensures PCFs in more applications. Besides, different shapes of the PCFs can be designed, e.g. hexagonal, non-hexagonal, octagonal, circular, decagonal, and hybrid cladding structured. [3-7].

In recent years, the design of PCF-SPR biosensors has attracted researcher's attention because of their applications in many areas, such as the analysis of biomolecular interactions (BIA) and precise detection of chemical and biological analytes

Received: 25 August 2018 / Revised: 10 October 2018

(C) The Author(s) 2018. This article is published with open access at Springerlink.com

DOI: $10.1007 / \mathrm{s} 13320-018-0524-7$

Article type: Regular 
[8]. For example, a PCF-SPR biosensor for the low refractive index (RI) detection was proposed by Liu et al. [9] with low spectral sensitivity and amplitude sensitivity of $6000 \mathrm{~nm} / \mathrm{RIU}$ and $600 \mathrm{RIU}^{-1}$, respectively. In the same fashion, side-polished hexagonal structured D-shaped PCF-SPR biosensor was demonstrated by Tiesheng et al. [10] with the excellent value of wavelength sensitivity (21 $700 \mathrm{~nm} / \mathrm{RIU}$ ) without considering amplitude sensitivity which is a very important term for characterizing the SPR sensor. Moreover, highly sensitive SPR biosensors were reported that focused on the high spectral sensitivity rather than considering amplitude sensitivity $[11,12]$.

The above reported SPR sensors are designed in a single core. However, the matured stack-and-draw fabrication technology makes it easier for fabricating multi-core PCFs, since multi-core PCFs including dual-core (DC) PCFs have exposed outstanding performances in different areas of applications, such as polarization splitter, polarization filter, and refractive index (RI) sensors [13-15]. After all, a number of dual core PCF biosensors were also reported in [16-18]. For example, a DC-SPR biosensor using silver-graphene as a plasmonic material with high spectral sensitivity of $10000 \mathrm{~nm} / \mathrm{RIU}$ and a high resolution of $1 \times 10^{-6} \mathrm{RIU}$ was reported by Wang et al. [16]. Also, DC-PCF sensor using gold as a plasmonic material with a high value of amplitude sensitivity but lower value of wavelength sensitivity was proposed by Paul et al. [17]. In a similar manner, DC square lattice PCF biosensor with excellent value of wavelength sensitivity (25000 nm/RIU) and lower value of amplitude sensitivity (about to $470 \mathrm{RIU}^{-1}$ ) was recommended for which gold was used as a plasmonic material [18].

In this article, we propose a DC-PCF refractive index sensor with excellent performance for which gold is used as a plasmonic material. The numerical examination is accomplished in both $x$ - and $y$-polarized modes in the broader sensing ranges from analyte RI of 1.33 to 1.41 . In the same way, the plasmonic material thickness and other structural parameters are optimized for getting the best performance.

\section{Methodology}

For investigating the guiding properties of the proposed PCF-SPR RI sensor, the finite element method (FEM) is used by allowing for perfectly matched layer (PML) boundary conditions as a radiation absorber [15]. The cross-sectional view of the proposed square lattice PCF RI sensor in the $x-y$ plane is shown in Fig. 1. The square lattice structure is chosen in such a way that it can increase the touch area between the core, and the annular gold layer-energy coupling between the plasmonic mode and the fundamental mode is stronger so that the sensitivity is increased [19].

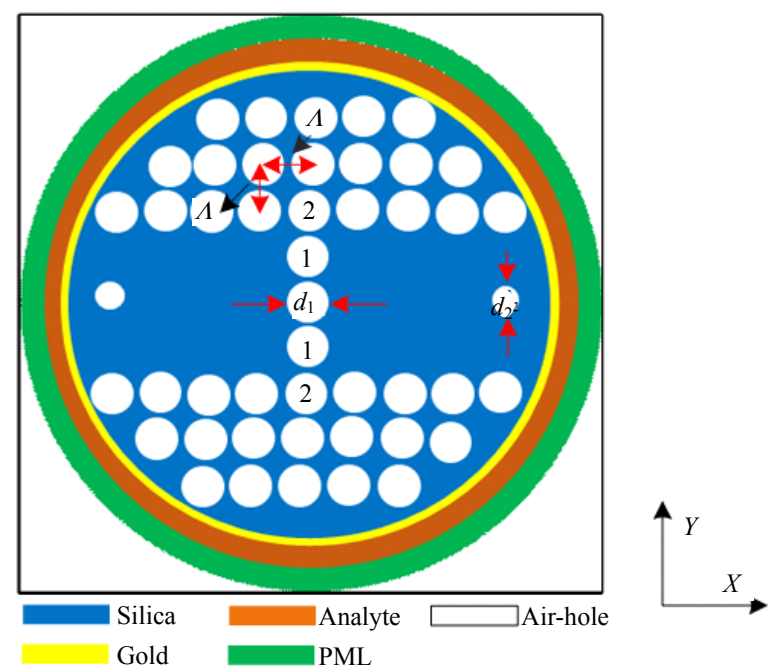

Fig. 1 Cross sectional view of the proposed RI sensor in $x-y$ plane.

As to the proposed sensor, the central air-hole (Fig. 1) is placed at the center with a diameter of $d_{1}=$ $0.8 \mu \mathrm{m}$. The air-hole number marked as 1 (Fig. 1) with the same diameter is placed at a distance (pitch) of $\Lambda=1 \mu \mathrm{m}$ from the central air-hole. Also, the air-hole number 2 with the same diameter is placed at a distance of $\Lambda$ from the air-hole number 1 . In this row, there are nine air-holes where each air-hole is separated at a distance of $\Lambda$ from the previous one. 
Similarly, the other air-holes in the other row are designed in the same way. The small air-holes with a diameter $d_{2}=0.4 \mu \mathrm{m}$ at two opposite sides of the outer ring are designed intentionally which are used to concentrate the evanescent field so that the surface plasmon can be excited efficiently. The background material is fused, silica and the plasmonic material is gold that has been characterized by Sellmeier equation and DrudeLorentz model, respectively [20].

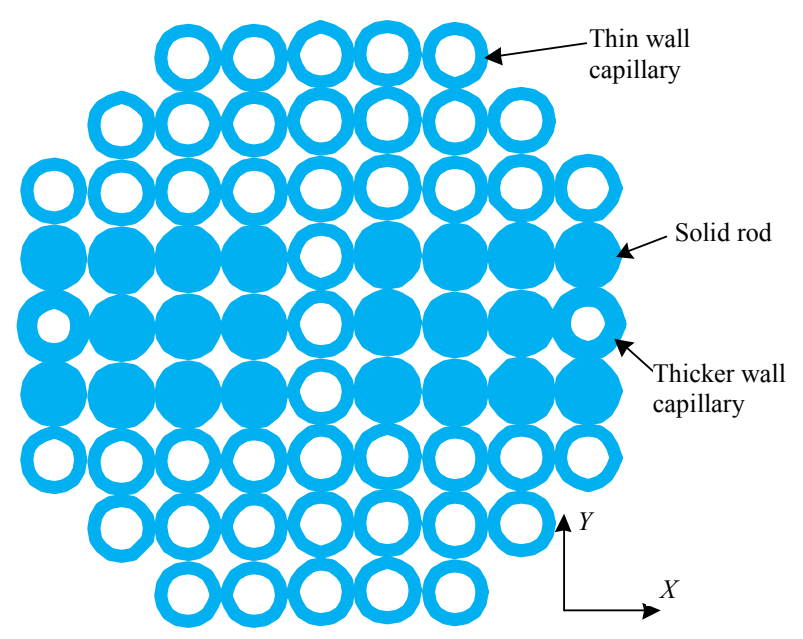

Fig. 2 Stacked preform of the proposed PCF in the $x-y$ plane.

The standard way of manufacturing PCF involves two main steps: fabrication of a fiber-preform and drawing by using a high temperature furnace in a standard fiber drawing tower. Firstly, various vapour deposition techniques are established for the fabrication of fiber performs, such as modified chemical vapour deposition (MCVD), vapour axial deposition (VAD), and outside vapour deposition (OVD) [21]. These techniques allow for the fabrication of preforms with silica glasses having very low, un-intentional, impurity levels, and precisely controlled doping levels. After the preparation of fiber performance, silica rods and capillaries are stacked together called stacked preform and drawn down by using a standard fiber drawing tower. Guiding cores are created by introducing deliberate defects in the lattice, for example by replacing a capillary with a solid rod, or leaving out several capillaries altogether [22, 23]. All the thinner wall capillary, thicker wall capillary, and solid rods are stacked together called stacked preform as shown in Fig. 2. After forming the stacked performance, the proposed DC-PCF can be fabricated by using fiber drawing tower.

\section{Results and discussion}

The optical field distribution of the proposed PCF-SPR RI sensor is shown in Fig. 3, and it can be seen that the strength of the electric field of surface plasmon polariton mode (SPP) is stronger for the $y$-polarized mode than that for the $x$-polarized mode. Therefore, the sensitivity will be greater for the $y$-polarized mode than that for the $x$-polarized mode.
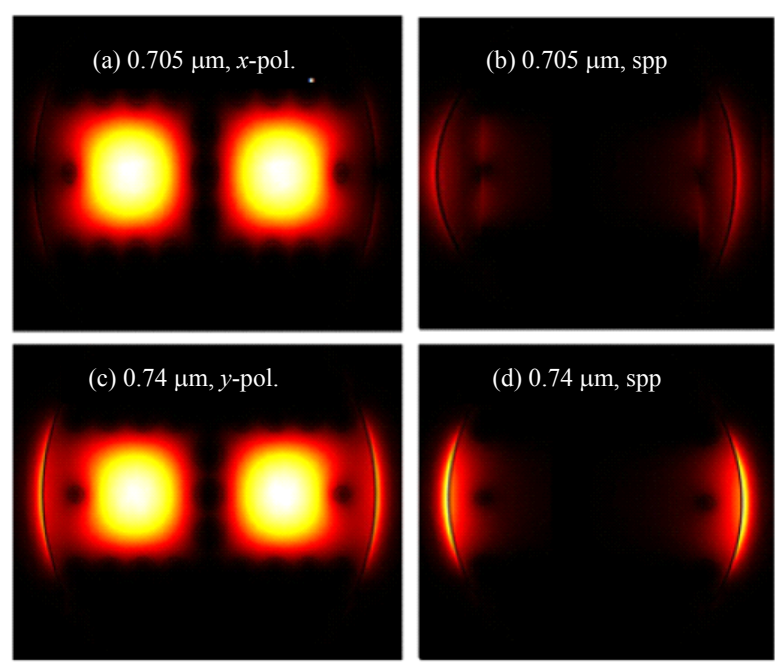

Fig. 3 Optical field distribution of the proposed biosensor: (a) $x$ - and (c) $y$-polarized fundamental modes and (b) $x$ - and (d) $y$-polarized SPP modes for analyte RI of 1.38 .

Figure 4 shows the dispersion relation between core-guided mode and SPP mode. At the wavelengths of $0.705 \mu \mathrm{m}$ and $0.74 \mu \mathrm{m}$, the confinement loss is the maximum $(5.899 \mathrm{~dB} / \mathrm{cm}$ and $154.7 \mathrm{~dB} / \mathrm{cm}$ ) for $x$ - and $y$-polarized modes, respectively. The corresponding wavelengths are called resonance wavelength as phase matching is done perfectly of these wavelength. 


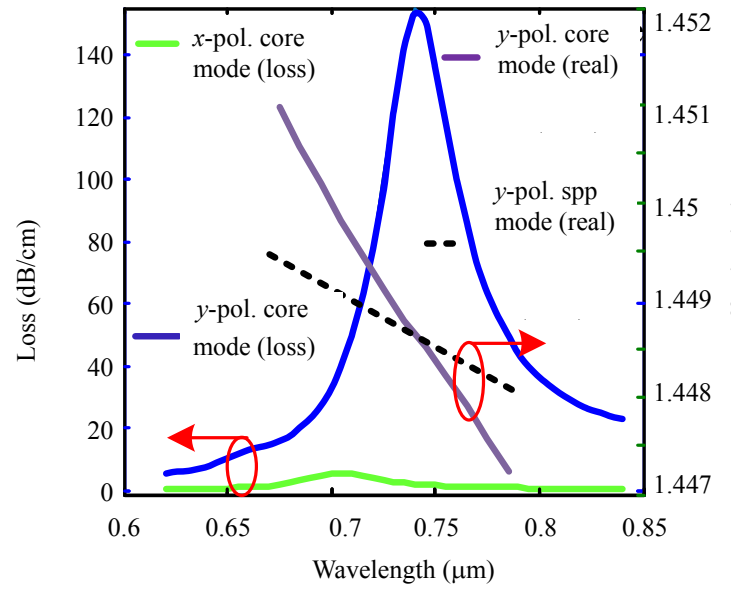

(a)

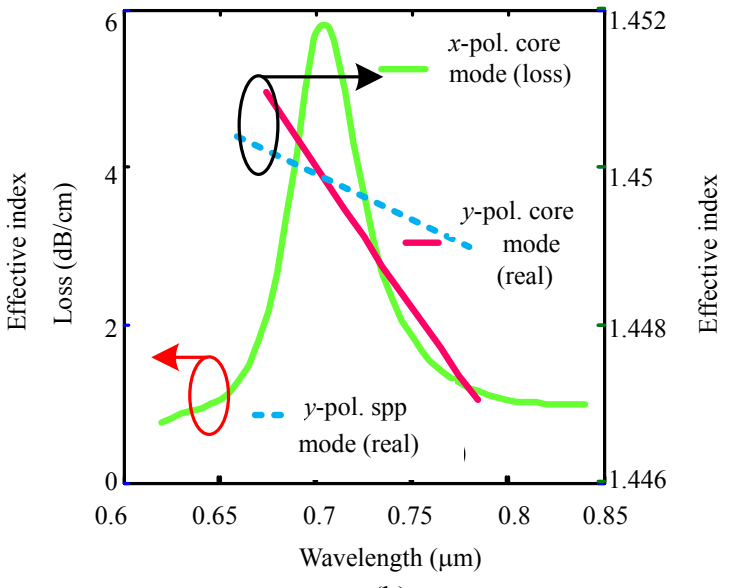

(b)

Fig. 4 Dispersion relationship of core guided and plasmonic modes for: (a) $y$-and (b) $x$-polarization modes for analyte RI of 1.38 .

\subsection{Wavelength sensitivity}

Wavelength sensitivity is measured for the change of the resonance wavelength per unit change in analyte RI. The resonance wavelength can be observed from the confinement loss of the proposed PCF sensor which could be calculated as [22]

$$
\alpha \approx 8.686 \times k_{0} \cdot \operatorname{Im}\left(n_{\text {eff }}\right) \times 10^{4}(\mathrm{~dB} / \mathrm{cm})
$$

where $n_{\text {eff }}$ is the imaginary part of the effective RI, and $k_{0}$ is the free space wave number. The loss is calculated by using the above equation shown in Fig. 5 which is the function of wavelength for different analyte RIs. For each analyte RI, the loss curve reaches its maximum value at the corresponding wavelength called resonance wavelength and it is gained to $0.6 \mu \mathrm{m}, 0.61 \mu \mathrm{m}, 0.63$ $\mu \mathrm{m}, 0.65 \mu \mathrm{m}, \quad 0.675 \mu \mathrm{m}, 0.705 \mu \mathrm{m}, 0.74 \mu \mathrm{m}, 0.782$ $\mu \mathrm{m}$, and $0.84 \mu \mathrm{m}$, for the analyte RIs of $1.33,1.34$, $1.35,1.36,1.37,1.38,1.39,1.40$, and 1.41, respectively, for the $x$-polarized mode and $0.61 \mu \mathrm{m}$, $0.63 \mu \mathrm{m}, 0.65 \mu \mathrm{m}, 0.675 \mu \mathrm{m}, 0.705 \mu \mathrm{m}, 0.74 \mu \mathrm{m}$, $0.793 \mu \mathrm{m}, 0.865 \mu \mathrm{m}$, and $0.98 \mu \mathrm{m}$, for the $y$-polarized mode.

The proposed sensor shows the resonance wavelength shifts of $10 \mathrm{~nm}, 20 \mathrm{~nm}, 20 \mathrm{~nm}, 25 \mathrm{~nm}$, $30 \mathrm{~nm}, 35 \mathrm{~nm}, 42 \mathrm{~nm}$, and $58 \mathrm{~nm}$ for corresponding analyte RIs of 1.33, 1.34, 1.35, 1.36, 1.37, 1.38, 1.39, and 1.40 , respectively, for the $x$-polarized mode. We use the wavelength interrogation method to measure the wavelength sensitivity stated as [23]:
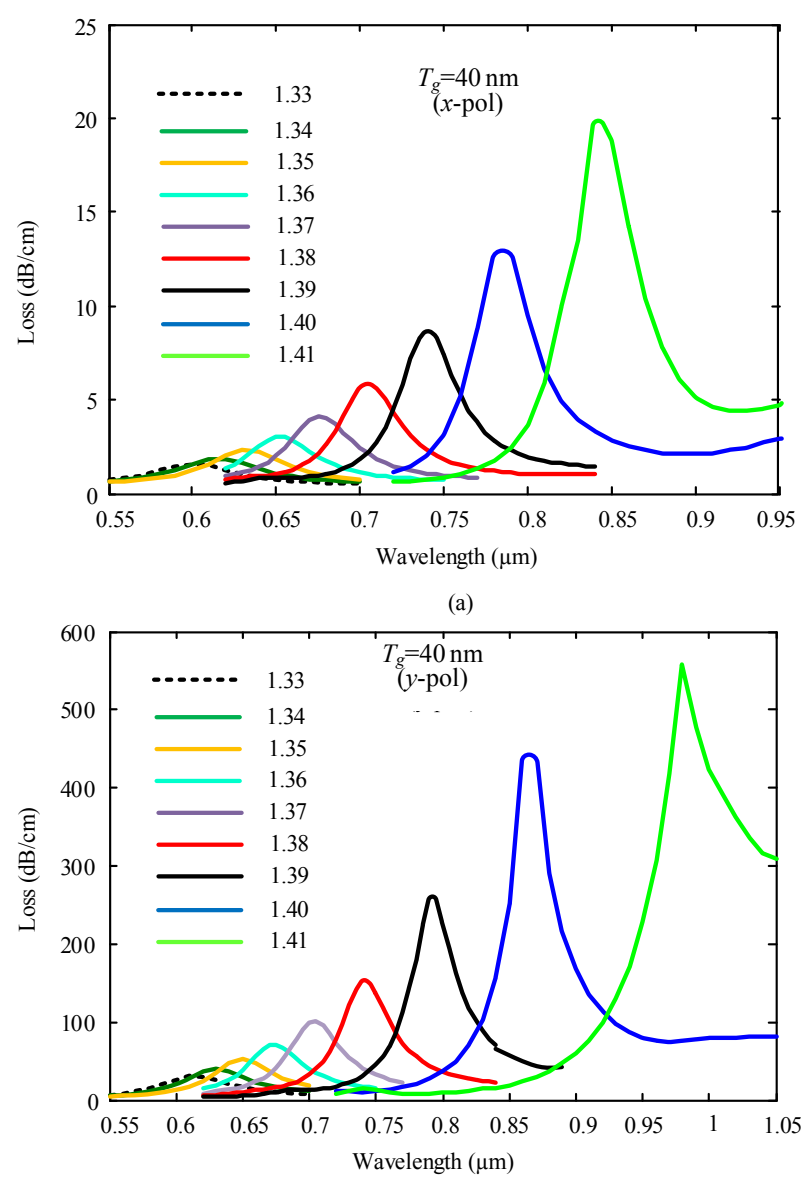

(b)

Fig. 5 Loss spectra as a function of wavelength for different analyte RIs for: (a) $x$ - and (b) $y$-polarized modes.

$$
S=\Delta \lambda_{\text {peak }} / \Delta n_{a}
$$

where $\Delta \lambda_{\text {peak }}$ and $\Delta n_{a}$ represent the resonance peak shift and change in analyte RIs, respectively. 
Therefore, the theoretical wavelength sensitivity is calculated using the above equation as $1000 \mathrm{~nm} / \mathrm{RIU}$, $2000 \mathrm{~nm} / \mathrm{RIU}, \quad 2000 \mathrm{~nm} / \mathrm{RIU}, 2500 \mathrm{~nm} / \mathrm{RIU}$, $3000 \mathrm{~nm} / \mathrm{RIU}, 3500 \mathrm{~nm} / \mathrm{RIU}, 4200 \mathrm{~nm} / \mathrm{RIU}$, and $5800 \mathrm{~nm} / \mathrm{RIU}$ for the analyte RIs of 1.33 to 1.40 , respectively.

In addition, the theoretical wavelength sensitivities are $2000 \mathrm{~nm} / \mathrm{RIU}, 2000 \mathrm{~nm} / \mathrm{RIU}$, $2500 \mathrm{~nm} / \mathrm{RIU}, \quad 3000 \mathrm{~nm} / \mathrm{RIU}, \quad 3500 \mathrm{~nm} / \mathrm{RIU}$, $5300 \mathrm{~nm} / \mathrm{RIU}, 7200 \mathrm{~nm} / \mathrm{RIU}$, and $11500 \mathrm{~nm} / \mathrm{RIU}$ for the $y$-polarized mode. The maximum wavelength sensitivity is achieved to $5800 \mathrm{~nm} / \mathrm{RIU}$, and $11,500 \mathrm{~nm} / \mathrm{RIU}$ for $x$ - and $y$-polarized modes, respectively, which are higher than the results reported in [18, 24, 25].

\subsection{Amplitude sensitivity}

A less complex and cost effective method, amplitude interrogation, is used to calculate the amplitude sensitivity [26] as

$$
S_{A}=-\left(1 / \alpha\left(\lambda, n_{a}\right)\right) \times\left(\Delta \alpha\left(\lambda, n_{a}\right) / \Delta n_{a}\right)
$$

where $\alpha\left(\lambda, n_{a}\right), \Delta \alpha\left(\lambda, n_{\mathrm{a}}\right)$, and $\Delta n_{a}$ indicate the loss of the core mode, differential loss between two consecutive analyte RIs, and the change in analyte RIs, respectively. Based on (3), the amplitude sensitivity is calculated and plotted with respect to the wavelength for both $x$ - and $y$-polarized modes as shown in Figs. 6(a) and 6(b), while analyte RI is changed from 1.33 to 1.40 . It can be evident from the figure that the amplitude sensitivity increases gradually with an increase in the analyte RI and reaches the maximum for analyte RI of 1.40 which are $554.9 \mathrm{RIU}^{-1}$ and $636.5 \mathrm{RIU}^{-1}$ for $x$ - and $y$-polarized modes, respectively, which are higher than the results reported in $[9,17,24,27,28]$.

\subsection{Sensor resolution}

The sensor resolution of the refractive index sensor is the smallest change that can be detected in the quantity it measures. Actually, the resolution is related to the precision of the measurement, but they are not the same thing, and it can be expressed as [29]

$$
R=\Delta n_{a} \times \Delta \lambda_{\text {min }} / \Delta \lambda_{\text {peak }} \text { (RIU) }
$$

where $\Delta \lambda_{\text {peak }}, \Delta \lambda_{\min }$, and $\Delta n_{a}$ indicate the resonance peak shift, the minimum spectral resolution, and the change in analyte RIs, respectively.

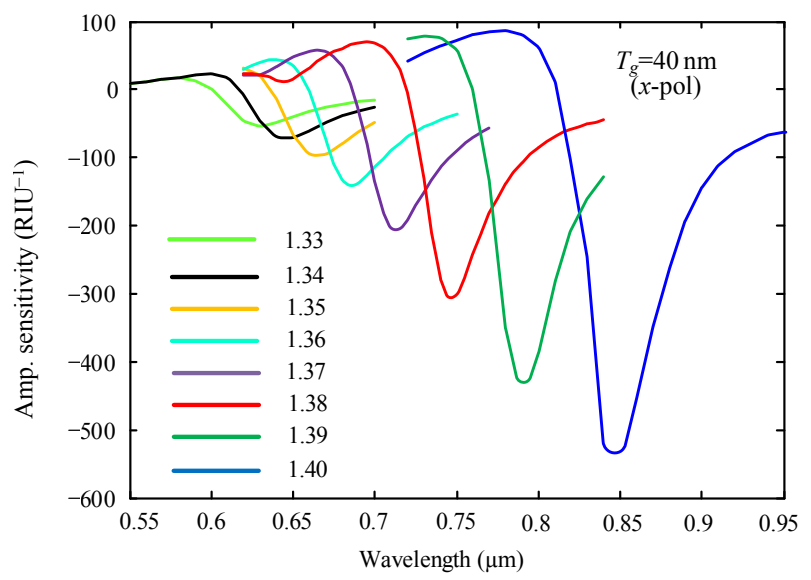

(a)

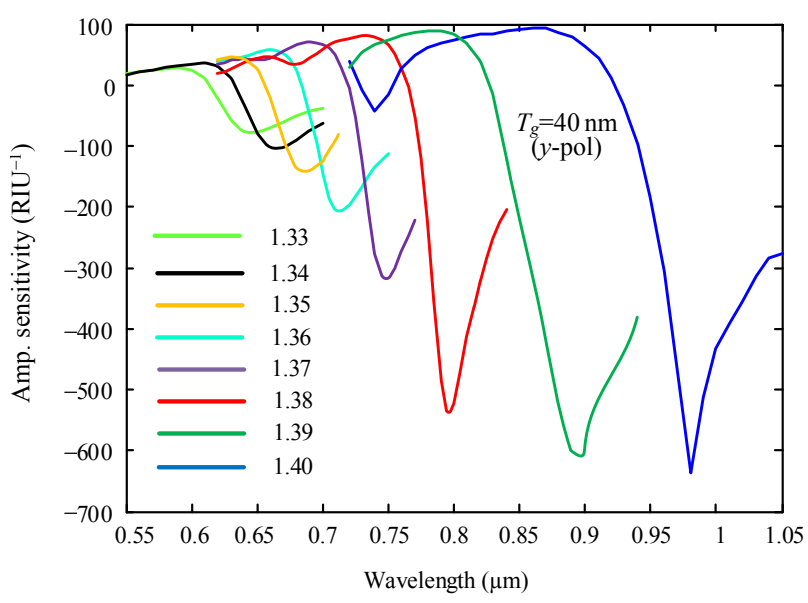

(b)

Fig. 6 Amplitude sensitivity as a function of wavelength for different analyte RIs for (a) $x$ - and (b) $y$-polarized modes.

According to (4), it is clear that the greater the resonance wavelength shift is, the lower the sensor resolution is, hence, the small amount of change in analyte RI can be detected precisely. The maximum sensor resolution of the proposed design is calculated as $1.72 \times 10^{-5}$ RIU and $8.7 \times 10^{-6}$ RIU for $x$ - and $y$-polarized modes, respectively for the analyte RI of 1.40 , which is higher than the resolution reported in [18, 27, 28].

Basically, the function of PCF based SPR sensor depends on the evanescent field. For plasmonic phenomenon, the main factor is to excite the free electrons of the metal surface efficiently and 
effectively [18]. In this design, dual core is introduced so that the evanescent field can face smaller distance to excite the free electrons of the plasmonic material as the nature of evanescent field is exponentially decayed with distance [17]. In addition, the sensitivity depends on the strength of the surface plasmon wave (SPW). If the single core is introduced, the evanescent field will face larger distance and tend to die out before reaching the metal surface and it will not be possible to get effective excitation of the free electrons. The design mechanism of our proposed sensor is such that the evanescent field can easily penetrate into the cladding region and interact with the free electrons of the metal surface. The electrons will start to resonate when phase matching occurs due to the resulted SPW generation. Also, the diameter of air-holes is smaller than that of other air-holes (located at the two opposite sides of the core), so the evanescent field gets an easy pathway to strike the metal surface, which increases the strength of SPW and enhances the sensitivity.

\section{Effect of gold layer thickness on sensitivity}

The reduction of the loss depth and red shift of the resonance wavelength occur with an increase in the gold layer thickness due to the damping effect of

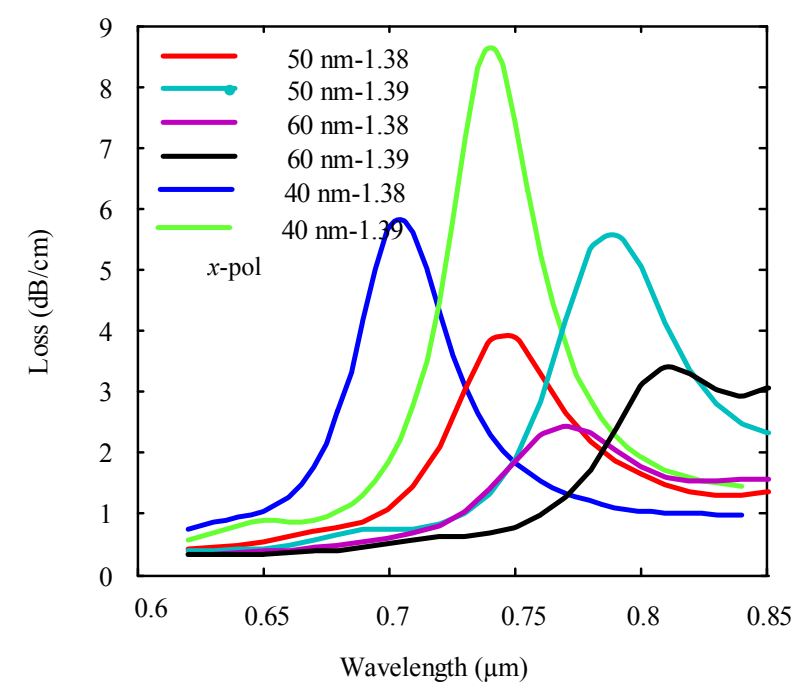

(a) the gold layer [27]. The reduction of loss depth results in weak interaction between the evanescent field and the analyte, hence reducing the amplitude sensitivity [17]. For example, we have varied the gold layer thicknesses to $40 \mathrm{~nm}, 50 \mathrm{~nm}$, and $60 \mathrm{~nm}$ for the analyte RIs of 1.38 and 1.39 .

When the gold layer thickness is increased, then the resonance wavelength is shifted towards the higher wavelength for the same analyte RI as well as a decrease in the loss depth, as shown in Fig. 7. In the case of the $x$-polarized mode, the wavelength sensitivity is achieved to $3500 \mathrm{~nm} / \mathrm{RIU}$, $3900 \mathrm{~nm} / \mathrm{RIU}$, and $4000 \mathrm{~nm} / \mathrm{RIU}$ for $t_{g}=40 \mathrm{~nm}$, $50 \mathrm{~nm}$, and $60 \mathrm{~nm}$, respectively, for analyte RI of 1.38. Besides, in the case of the $y$-polarized mode, the wavelength sensitivities of $5300 \mathrm{~nm} / \mathrm{RIU}$, $5900 \mathrm{~nm} / \mathrm{RIU}$, and $6000 \mathrm{~nm} / \mathrm{RIU}$ are gained for the same gold layer thickness. Actually, the gold layer thickness has a major impact on the amplitude sensitivity, and it is calculated as $308.5 \mathrm{RIU}^{-1}$, 205.9 $\mathrm{RIU}^{-1}$, and $116.3 \mathrm{RIU}^{-1}$ for the analyte RI of 1.38 for $t_{g}=40 \mathrm{~nm}, 50 \mathrm{~nm}$, and $60 \mathrm{~nm}$, respectively, for $x$-polarized mode and $546 \mathrm{RIU}^{-1}, 349.5 \mathrm{RIU}^{-1}$, and $178.5 \mathrm{RIU}^{-1}$ for $y$-polarized mode as shown in Fig. 8. Therefore, the gold layer thickness is chosen as $40 \mathrm{~nm}$ for getting the highest degree of sensing performance.

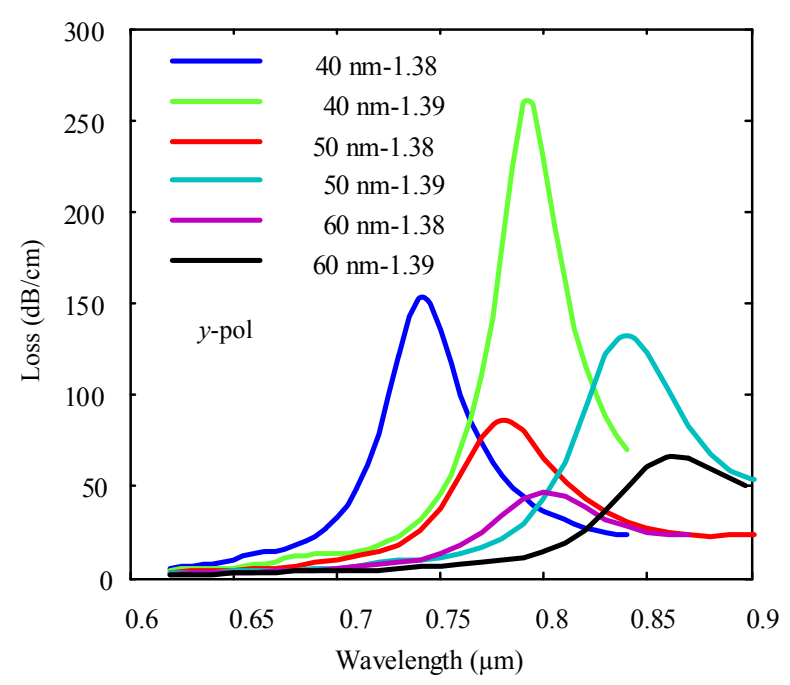

(b)

Fig. 7 Loss spectra as a function of wavelength with different gold layer thicknesses for: (a) $x$ - and (b) $y$-polarized modes. 


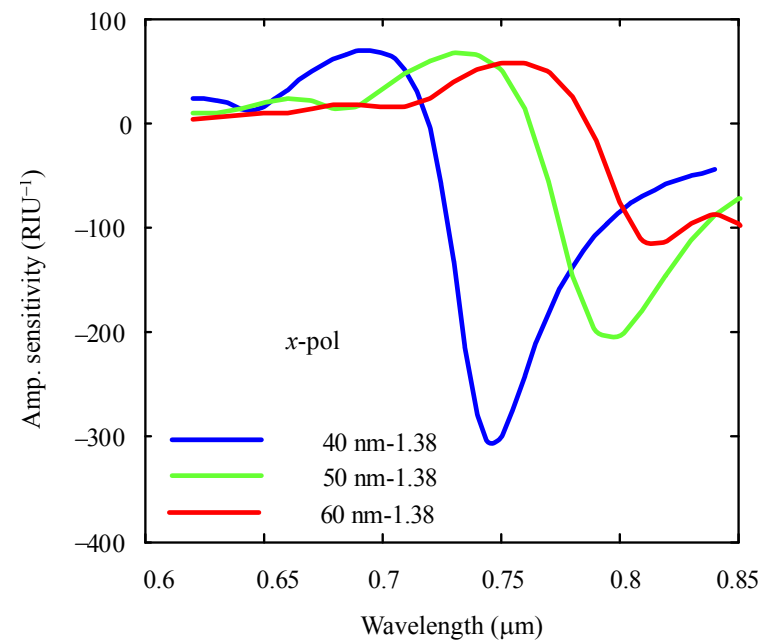

(a)

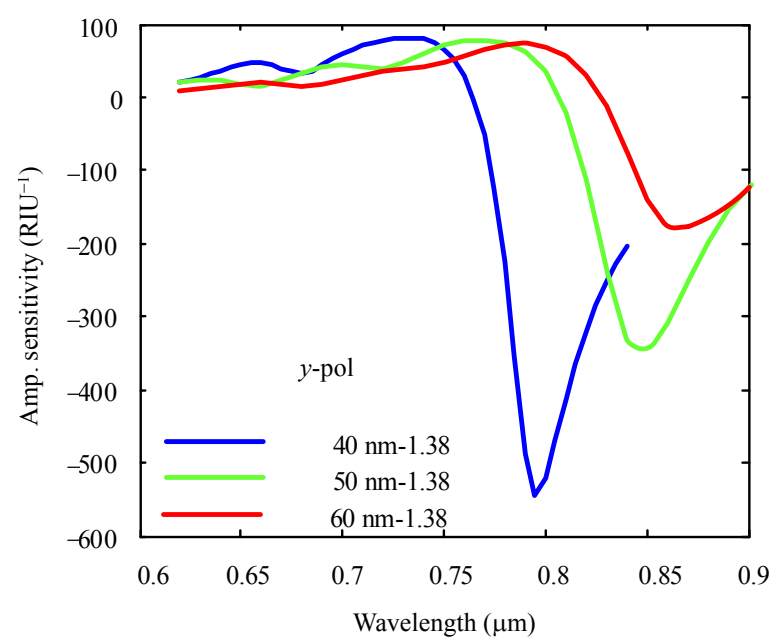

(b)

Fig. 8 Amplitude sensitivity as a function of wavelength with different gold layer thicknesses for: (a) $x$ - and (b) $y$-polarized modes.

\section{Effect of the variation of structural parameters on sensitivity}

After obtaining the optimum performance of the proposed sensor to a desired level, the performance accuracy of the proposed refractive index sensor is checked by varying $d_{1}, d_{2}$ and $\Lambda$ of $\pm 2 \%$ from their optimum value for the analyte RIs of 1.38 and 1.39, which are shown in Figs. 9 to 11. Figures. 9 and 10

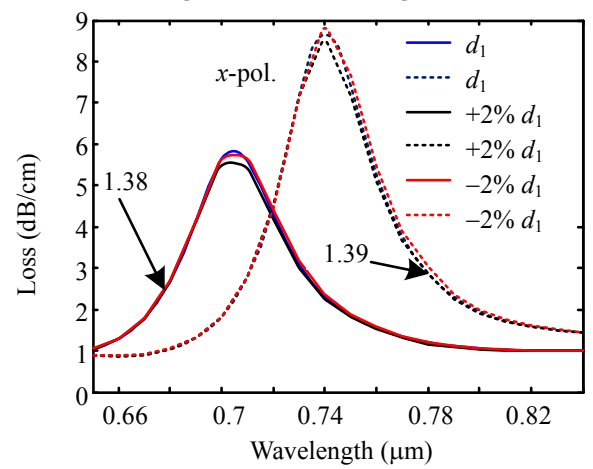

(a)

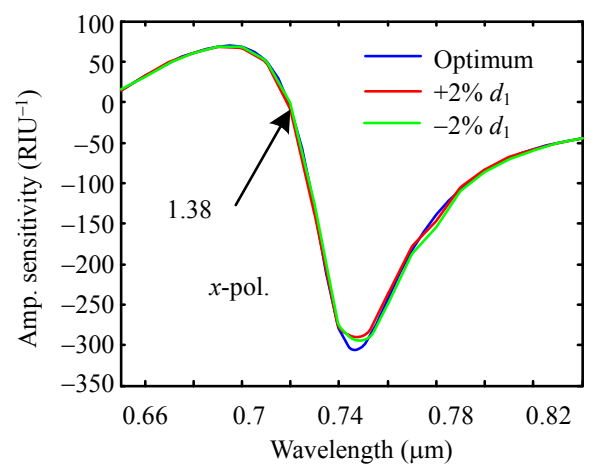

(c) show the loss spectra and amplitude sensitivity as a function of wavelength where the loss depth reduces with the variation of $d_{1}$ and $d_{2}$ by $\pm 2 \%$ from its optimum value because of the reduction of the effective index difference between core and cladding, hence, it reduces the amplitude sensitivity both for $x$ - and $y$-polarized modes.

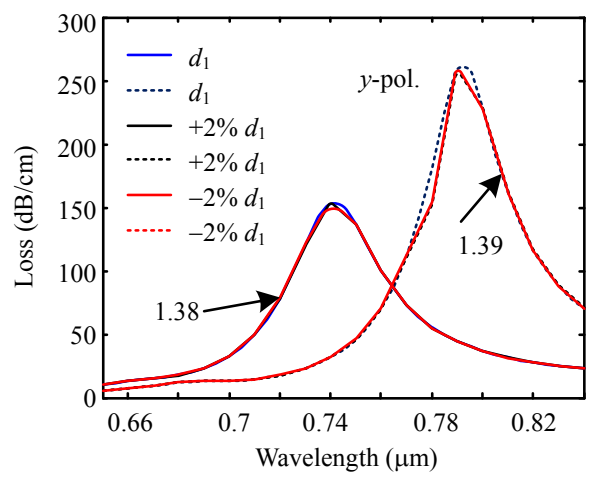

(b)

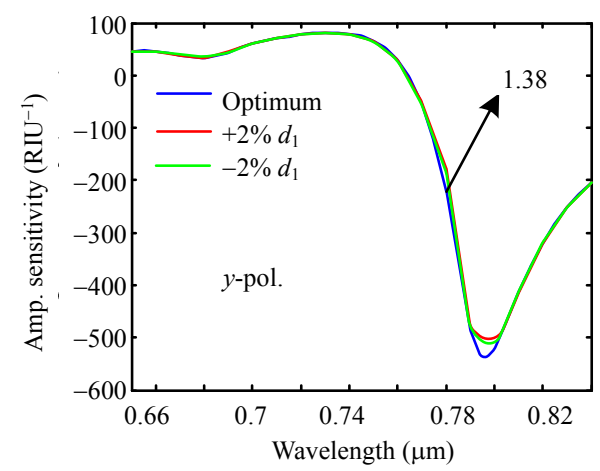

(d)

Fig. 9 Loss spectra as a function of wavelength for: (a) $x$ - and (b) $y$-polarized modes, amplitude sensitivity as a function of wavelength for (c) $x$ - and (d) $y$-polarized modes while $d_{1}$ is changed by $\pm 2 \%$. 


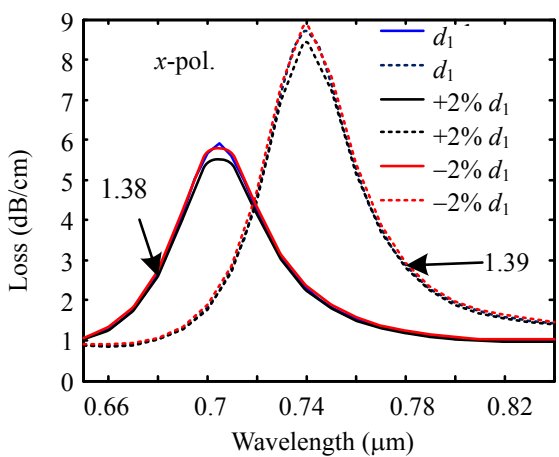

(a)

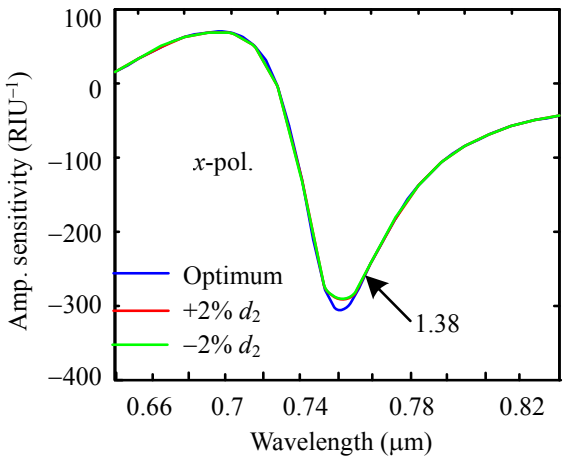

(c)

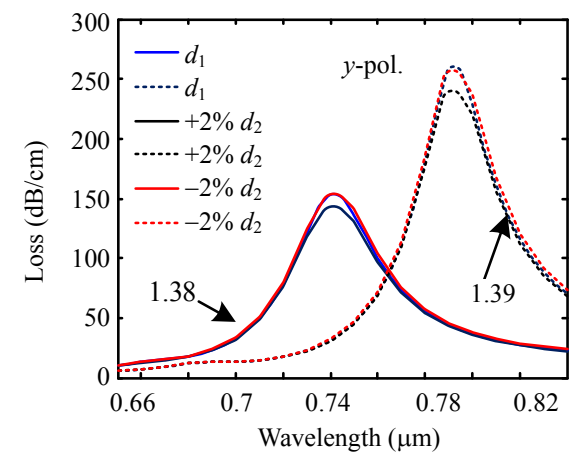

(b)

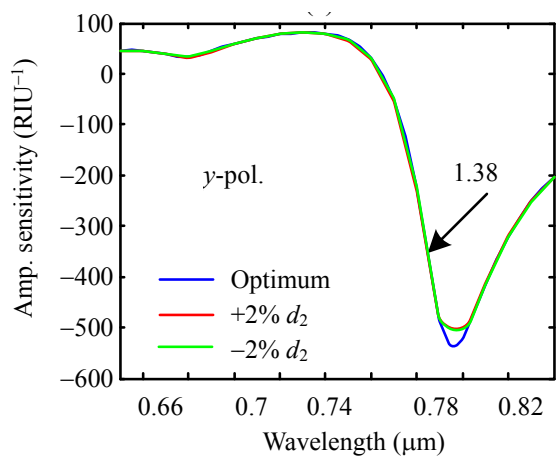

(d)

Fig. 10 Loss spectra as a function of wavelength for: (a) $x$ - and (b) $y$-polarized modes, amplitude sensitivity as a function of wavelength for (c) $x$ - and (d) $y$-polarized modes while $d_{2}$ is changed by $\pm 2 \%$.

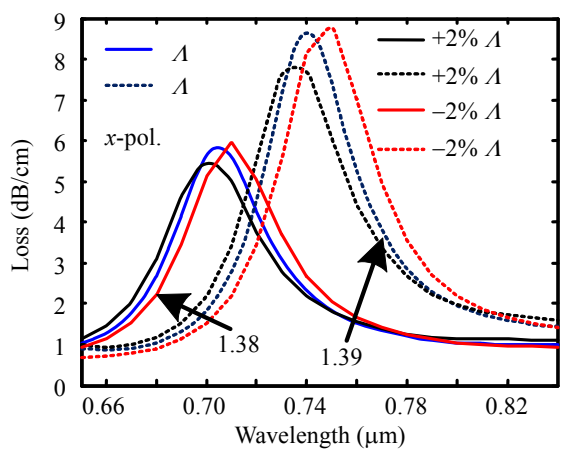

(a)

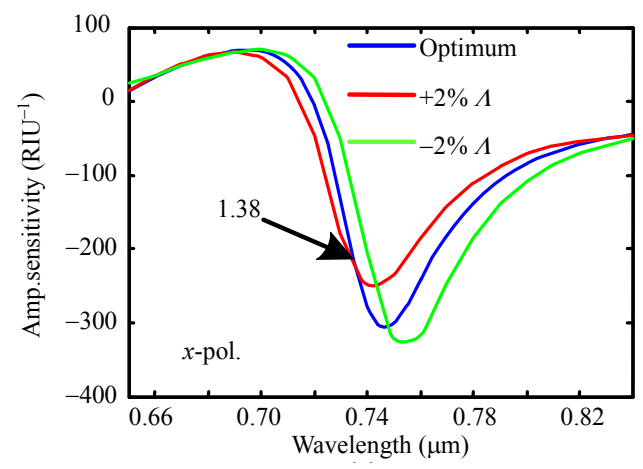

(c)

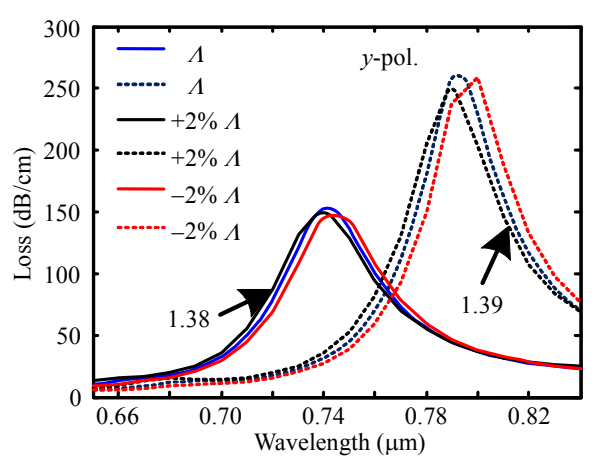

(b)

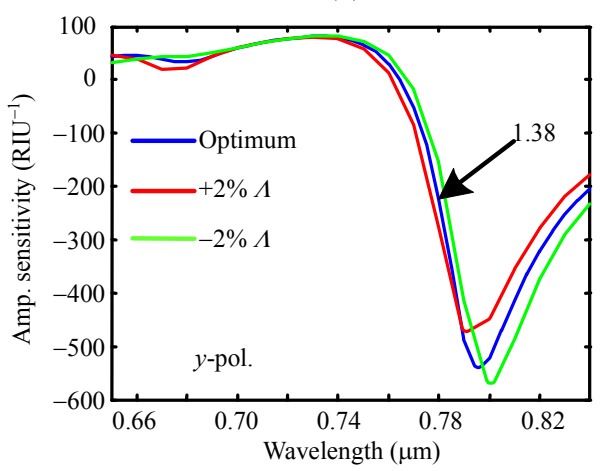

(d)

Fig. 11 Loss spectra as a function of wavelength for: (a) $x$ - and (b) $y$-polarized modes, amplitude sensitivity as a function of wavelength for (c) $x$ - and (d) $y$-polarized modes while $\Lambda$ is changed by $\pm 2 \%$. 
Furthermore, Fig. 11 shows the loss spectra and amplitude sensitivity curve for $\Lambda$ variation by $\pm 2 \%$. If $\Lambda$ is varied by $+2 \%$, the difference of $n_{\text {eff }}$ between core and cladding increases, hence, the loss depth is reduced both for $x$ - and $y$-polarized modes, then the amplitude sensitivity reduces. Again, the loss depth increases with a decrease in $\Lambda$ by $-2 \%$ due to the reduction of $n_{\text {eff }}$ between the core and cladding both for $x$ - and $y$-polarized modes, then the amplitude sensitivity increases as shown in Fig. 11.

According to the above analysis, the proposed device structure shows acceptable fabrication tolerance as discussed by different authors [13, 30]. For instance, Reeve et al. [30] proposed a fabricated PCF with a fabrication tolerance of $\pm 1 \%$.

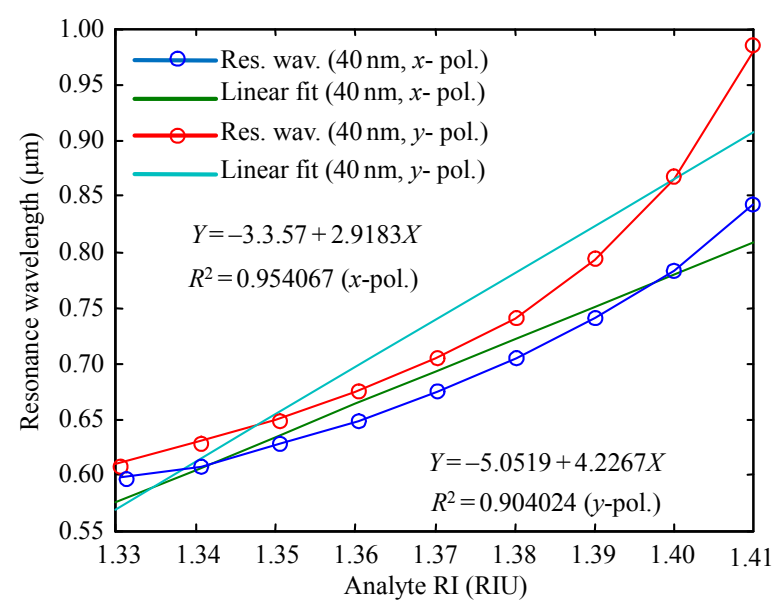

Fig. 12 Linear fitting of the resonance wavelength as a function of analyte RI.

A linear fitting characteristic of the resonance wavelength has a major impact on average sensitivity and resolution. Though the relationship between the resonance wavelength and the analyte RI is nonlinear, a linear fitting characteristic is very important for the optimization of the sensor in a defined dynamic range. High sensor linearity is a prerequisite for the calibration of sensors, especially for high RI analytes [17]. Moreover, the slope of the linear fitting curve represents the average sensitivity of a sensor. Basically, the linear fitting characteristic reduces the average sensitivity and resolution. High linearity indicates that the sensor shows high average sensitivity and resolution which make the sensor flexible for correctness measurement of the analytes. Nonlinearity of the response is not expected, as it creates significant distinction of the average sensitivity and resolution [17, 27]. The linear fitting equations are given in the inset of Fig. 12 and calculated co-efficient of the determination $\left(R^{2}\right)$ of the proposed sensor are 0.954067 and 0.904024 for $x$ - and $y$-polarized modes, respectively, which shows high linearity.

\section{Conclusions}

In this paper, a highly sensitive refractive index sensor based on DC-PCF has been analyzed through FEM based software. Numerical results show that our proposed sensor can detect a range of RIs from 1.33 to 1.41 . The highest wavelength sensitivity of $5800 \mathrm{~nm} / \mathrm{RIU}$ and $11500 \mathrm{~nm} / \mathrm{RIU}$ are proposed when the RI of the analyte is 1.40 for $x$ - and $y$-polarized modes, respectively. In addition, the maximum amplitude sensitivities of $554.9 \mathrm{RIU}^{-1}$ and 636.5 $\mathrm{RIU}^{-1}$ have been achieved for $x$ - and $y$-polarized modes, respectively. Therefore, considering its simple manufacturing process, our proposed sensor is a competitive candidate for the detection of biological and biochemical analytes.

\section{Acknowledgement}

The authors thank Dr. Abdul KHALEQUE for his valuable discussions and contributions for preparing the manuscript.

Open Access This article is distributed under the terms of the Creative Commons Attribution 4.0 International License (http://creativecommons.org/licenses/by/4.0/), which permits unrestricted use, distribution, and reproduction in any medium, provided you give appropriate credit to the original author(s) and the source, provide a link to the Creative Commons license, and indicate if changes were made.

\section{References}

[1] M. Y. Azab, M. F. O. Hameed, A. Heikal, M. A. Swillam, and S. Obayya, "Analysis of highly sensitive surface plasmon photonic crystal fiber biosensor," 
SPIE, 2018, 10541: 105411N-1-105411N-6.

[2] F. Wang, Z. Sun, C. Liu, T. Sun, and P. K. Chu, "A high-sensitivity photonic crystal fiber (PCF) based on the surface plasmon resonance (SPR) biosensor for detection of density alteration in non-physiological cells (DANCE)," Opto-Electronics Review, 2018, 26(1): 50-56.

[3] T. A. Birks, J. C. Knight, and P. S. J. Russell, "Endlessly single-mode photonic crystal fiber," Optics Letters, 1997, 22(13): 961-963.

[4] S. M. A. Razzak, Y. Namihira, M. A. Hossain, and A. Khaleque, "Designing birefringence of index-guiding non-hexagonal photonic crystal fibers," Journal of Optics, 2011, 40(2): 56-64.

[5] A. Khaleque and H. T. Hattori, "Polarizer based upon a plasmonic resonant thin layer on a squeezed photonic crystal fiber," Applied Optics, 2015, 54(9): 2543-2549.

[6] J. Knight, J. Arriaga, T. Birks, A. O. Blanch, W. Wadsworth, and P. S. J. Russell, "Anomalous dispersion in photonic crystal fiber," IEEE Photonics Technology Letters, 2000, 12(7): 807-809.

[7] W. J. Wadsworth, N. Joly, J. C. Knight, T. A. Birks, F. Biancalana, and P. S. J. Russell, "Supercontinuum and four-wave mixing with Q-switched pulses in endlessly single-mode photonic crystal fibres," Optics Express, 2004, 12(2): 299-309.

[8] C. Liu, W. Q. Su, Q. Liu, X. L. Lu, F. M. Wang, T. Sun, et al., "Symmetrical dual D-shape photonic crystal fibers for surface plasmon resonance sensing," Optics Express, 2018, 26(7): 9039-9049.

[9] C. Liu, L. Yang, Q. Liu, F. M. Wang, Z. J. Sun, T. Sun, et al., "Analysis of a surface plasmon resonance probe based on photonic crystal fibers for low refractive index detection," Plasmonics, 2018, 13(3): 779-784.

[10] T. S. Wu, Y. Shao, Y. Wang, S. Q. Cao, W. P. Cao, F. Zhang, et al., "Surface plasmon resonance biosensor based on gold-coated side-polished hexagonal structure photonic crystal fiber," Optics Express, 2017, 25(17): 20313-20322.

[11] C. Liu, L. Yang, W. Q. Su, F. M. Wang, T. Sun, Q. Liu, et al., "Numerical analysis of a photonic crystal fiber based on a surface plasmon resonance sensor with an annular analyte channel," Optics Communications, 2017, 382: 162-166.

[12]S. Chakma, M. A. Khalek, B. K. Paul, K. Ahmed, M. R. Hasan, and A. N. Bahar, "Gold-coated photonic crystal fiber biosensor based on surface plasmon resonance: design and analysis," Sensing and Bio-Sensing Research, 2018, 18: 7-12.

[13] A. Khaleque and H. T. Hattori, "Ultra-broadband and compact polarization splitter based on gold filled dual-core photonic crystal fiber," Journal of Applied Physics, 2015, 118(14): 682-683.

[14] W. Zhang, S. Q. Lou, and X. Wang, “A polarization filter based on a novel photonic crystal fiber with a gold-coated air hole by using surface plasmon resonance," Plasmonics, 2018, 13(2): 365-371.

[15] Y. X. Liu, S. P. Zhan, G. T. Cao, H. Yang, J. Li, Q. Liu, et al., "Theoretical design of plasmonic refractive index sensor based on the fixed band detection," IEEE Journal of Selected Topics in Quantum Electronics, 2018, 25(2): 1-1.

[16] F. Wang, Z. Sun, C. Liu, T. Sun, and P. K. Chu, "A highly sensitive dual-core photonic crystal fiber based on a surface plasmon resonance biosensor with silver-graphene layer," Plasmonics, 2017, 12(6): 1847-1853.

[17] A .K. Paul, A. K. Sarkar, M. A. B. S. Rahman, and A. Khaleque, "Twin core photonic crystal fiber plasmonic refractive index sensor," IEEE Sensors Journal, 2018, 18(14): 5761-5769.

[18] A. K. Paul, A. K. Sarkar, M. H. Islam, and M. Morshed, "Dual core photonic crystal fiber based surface plasmon resonance biosensor," Optik-International Journal for Light and Electron Optics, 2018, 170: 400-408.

[19] G. W. An, S. G. Li, X. Yan, X. N. Zhang, Z. Y. Yuan, and Y. N. Zhang, "High-sensitivity and tunable refractive index sensor based on dual-core photonic crystal fiber," Journal of the Optical Society of America B, 2016, 33(7): 1330-1334.

[20] K. Tong, F. C. Wang, M. T. Wang, P. Dang, Y. X. Wang, and J. R. Sun, "D-shaped photonic crystal fiber biosensor based on silver-graphene," Optik-International Journal for Light and Electron Optics, 2018, 168: 467-474.

[21] A. Bjarklev, J. Broeng, and A. S. Bjarklev, Fabrication of photonic crystal fibres, photonic crystal fibres. Boston, USA: Springer, 2003: $115-130$.

[22]A. A. Rifat, F. Haider, R. Ahmed, G. A. Mahdiraji, F. R. M. Adikan, and A. E. Miroshnichenko, "Highly sensitive selectively coated photonic crystal fiber-based plasmonic sensor," Optics Letters, 2018, 43(4): 891-894.

[23] D. J. J. Hu and H. P. J. Hu, "Photonics, recent advances in plasmonic photonic crystal fibers: design, fabrication and applications," Advances in Optics and Photonics, 2017, 9(2): 257-314.

[24] M. R. Hasan, S. Akter, M. S. Rahman, and K. Ahmed, "Design of a surface plasmon resonance refractive index sensor with high sensitivity," Optical Engineering, 2017, 56(8): 087101-1087101-6.

[25] S. I. Azzam, M. F. O. Hameed, R. E. A. Shehata, A. Heikal, and S. S. A. Obayya, "Multichannel photonic crystal fiber surface plasmon resonance based sensor," Optical and Quantum Electronics, 2016, 48(2): 1-11.

[26] A. E. Khalil, A. H. E. Saeed, M. A. Ibrahim, M. E. Hashish, M. R. Abdelmonem, M. F. O. Hameed, et al., "Highly sensitive photonic crystal fiber 
biosensor based on titanium nitride," Optical and Quantum Electronics, 2018, 50(3): 158-1-158-12.

[27] M. R. Hasan, S. Akter, A. A. Rifat, S. Rana, K. Ahmed, R. Ahmed, et al., "Spiral photonic crystal fiber-based dual-polarized surface plasmon resonance biosensor," IEEE Sensors Journal, 2017, 18(1): 133-140.

[28] M. R. Hasan, S. Akter, A. A. Rifat, S. Rana, and S. Ali, "A highly sensitive gold-coated photonic crystal fiber biosensor based on surface plasmon resonance," Photonics, 2017, 4(1): 1-11.
[29] A. K. Paul, A. K. Sarkar, and S. A. Razzak, "Graphene coated photonic crystal fiber biosensor based on surface plasmon resonance," in Proceeding of 2017 IEEE Region 10 Humanitarian Technology Conference (R10-HTC), Dhaka, Bangladesh, 2017, pp. 856-859.

[30] W. H. Reeves, J. Knight, P. S. J. Russell, and P. Roberts, "Demonstration of ultra-flattened dispersion in photonic crystal fibers," Optics Express, 2002, 10(14): 609-613. 